\title{
Characterising claw-free t-perfect graphs
}

\author{
Maya Stein \\ Centro de Modelamiento Matemático, Universidad de Chile, Santiago, Chile, \\ email: <mstein@dim.uchile.cl> \\ Henning Bruhn \\ Mathematisches Seminar, Universität Hamburg, Germany, \\ email: <bruhn@math.uni-hamburg.de>
}

\begin{abstract}
We characterise the class of all claw-free $t$-perfect graphs by forbidden $t$-minors. Moreover, we show that claw-free $t$-perfect graphs are 3 -colourable. Such a colouring can be obtained in polynomial time.

Keywords: $t$-perfect graphs, claw-free graphs, stable set polytope (SSP), colouring.

\section{Introduction}

\section{1 t-perfect graphs}

It is well-known that perfect graphs can be determined by the structure of their stable set polytope. For a graph $G$, the stable set polytope, or $\operatorname{SSP}(G)$ for short, is the convex hull in $\mathbb{R}^{V}$ of the characteristic vectors of independent vertex sets, the stable sets. In the case of a perfect graph, the SSP is fully described by non-negativity and clique inequalities. Vice versa, if the SSP of some graph is given by these types of inequalities then the graph is perfect.
\end{abstract}


In analogy to this relationship, Chvátal [6] proposed to investigate a class of graphs now called $t$-perfect: the class of those graphs whose SSP is determined by non-negativity, edge and odd-cycle inequalities. More formally, let us define for a graph $G$ the polytope $\operatorname{TSTAB}(G) \subseteq \mathbb{R}^{V}$, given by

$$
\begin{gathered}
x \geq 0, \\
x_{u}+x_{v} \leq 1 \text { for every edge } u v \in E, \\
x(C) \leq\lfloor|C| / 2\rfloor \text { for every induced odd cycle } C \text { in } G .
\end{gathered}
$$

Clearly, it holds that $\operatorname{SSP}(G) \subseteq \operatorname{TSTAB}(G)$, and we say that the graph $G$ is $t$-perfect if $\operatorname{SSP}(G)$ and $\operatorname{TSTAB}(G)$ coincide. (Equivalently, $G$ is $t$-perfect if and only if $\operatorname{TSTAB}(G)$ is an integral polytope, i.e. if all its vertices are integral vectors.)

Neither the complete graph on four vertices $K_{4}$ nor the 5 -wheel $W_{5}$ are $t$-perfect. Indeed, for $K_{4}$ the vector $\left(\frac{1}{3}, \frac{1}{3}, \frac{1}{3}, \frac{1}{3}\right)$ lies in TSTAB but not in the SSP of $K_{4}$ as the sum of over all entries is larger than $\alpha\left(K_{4}\right)=1$. As for $W_{5}$, the vector that assigns a value of $\frac{2}{5}$ to each vertex on the rim and a value of $\frac{1}{5}$ to the centre shows that 5 -wheel is $t$-imperfect. Again, the vector lies in TSTAB but the sum of all entries is larger than $\alpha\left(W_{5}\right)=2$.

The class of $t$-perfect graphs includes the series-parallel graphs (Boulala and Uhry [2]), the bipartite graphs, and the almost bipartite graphs, i.e. those graphs that become bipartite upon deletion of a single vertex (Fonlupt and Uhry [8]). Gerards and Shepherd [9] characterise the graphs with all subgraphs $t$-perfect.

A graph is claw-free if it does not contain a claw, i.e. an induced $K_{1,3}$. We prove two theorems for claw-free $t$-perfect graphs. We characterise them in terms of forbidden substructures and we show that these graphs can be 3-coloured.

\subsection{Characterisation in terms of forbidden t-minors}

The celebrated strong perfect graph theorem of Chudnovsky, Robertson, Seymour and Thomas [5] characterises perfect graphs in terms of forbidden induced subgraphs: a graph is perfect if and only if it does not contain odd holes or anti-holes. We prove an analogous, although much more modest, result for claw-free $t$-perfect graphs. While, in order to describe perfect graphs, induced subgraphs are suitable as forbidden substructures, for $t$-perfect graphs a more general type of substructure, called a $t$-minor, is more appropriate.

A $t$-minor is any graph obtained from the original graph by (possibly repeated) application of (one or both of) two kinds of operations. The first of 
these is vertex deletion, and the second is t-contraction, that is, the simultaneous contraction of all edges incident with a vertex whose neighbourhood forms an independent set. Both operations preserve $t$-perfection (for vertex deletion this is obvious, for $t$-contraction see [9]). Thus $t$-minors inherit $t$-perfection, and we call a graph minimally $t$-imperfect if it is not $t$-perfect but every proper $t$-minor of it is $t$-perfect. Obviously, in order to characterise $t$-perfect graphs in terms of forbidden $t$-minors it suffices to find all minimally $t$-imperfect graphs.

We present all minimally $t$-imperfect claw-free graphs (there are four). As the property of being claw-free is preserved under $t$-contractions (which is easily verified), and clearly under vertex deletions, we thus characterise the class of all claw-free $t$-perfect graphs.

Theorem 1.1 A claw-free graph is t-perfect if and only if it does not contain any of $K_{4}, W_{5}, C_{7}^{2}$ and $C_{10}^{2}$ as a t-minor.
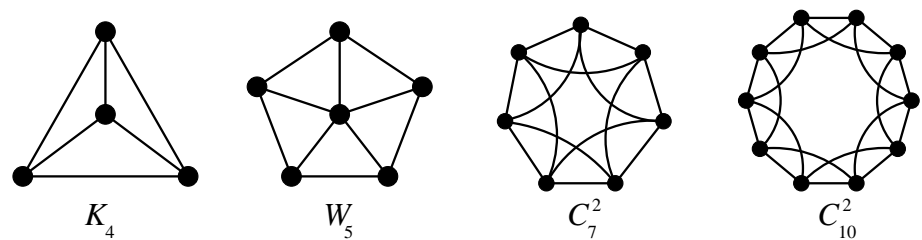

Fig. 1. The forbidden $t$-minors for claw-free graphs: the complete graph on four vertices, the 5 -wheel, the squares of the 7 - and the 10 -cycle.

\subsection{Colourings}

Standard polyhedral methods assert that the fractional chromatic number of a $t$-perfect graph is at most 3 . Shepherd suggested that $t$-perfect graphs might always be $k$-colourable for some fixed small $k$. As Laurent and Seymour gave an example of a $t$-perfect graph with $\chi=4$ (see [12, p. 1207]), this number $k$ has to be at least 4 .

Conjecture 1.2 Every t-perfect graph is 4-colourable.

We prove that for claw-free graphs three colours suffice.

Theorem 1.3 Every claw-free t-perfect graph is 3-colourable.

Moreover, from our proof it follows that every claw-free $t$-perfect graph on $n$ vertices can be coloured with three colours in polynomial time in $n$.

We remark that compared to a result of Chudnovsky and Ovetsky [4] our Theorem 1.3 yields an improvement of 1 . Indeed, Chudnovsky and Ovetsky 
show that the chromatic number of a quasi-line graph $G$ is bounded by $\frac{3}{2} \omega(G)$. As no $t$-perfect graph can contain a clique of at least four vertices and, furthermore, as a claw-free $t$-perfect graph is quasi-line, Chudnovsky and Ovetsky's bound is applicable and yields $\chi \leq 4$ for all claw-free $t$-perfect graphs.

Our result can be used for determining the chromatic number of $h$-perfect graphs, which have been introduced by Sbihi and Uhry [11] as a common generalisation of perfect and $t$-perfect graphs. For the definition of $h$-perfect graphs we use the same inequalities as for $t$-perfect graphs, only that the edge inequalities are replaced with clique inequalities. Then, an argment due to Sebő[personal communication] together with Theorem 2 yields the following.

Corollary 1.4 Let $G$ be a claw-free h-perfect graph. Then $\chi(G)=\left\lceil\chi^{*}(G)\right\rceil$ and $\chi(G)=\omega(G)$ if $\omega(G) \geq 3$.

\section{Proofs of our theorems}

We give an outline of our proofs. All details can be found in [3].

\subsection{The structure lemma}

The structure of a claw-free $t$-perfect graph is rather restricted. Already in a claw-free and $K_{4}$-free graph we observe a certain behaviour of the neighbourhoods. A vertex of such a graph neither may have three neighbours which form a stable set, nor three neighbours which induce a triangle. So, for example, the neighbourhood of a vertex of degree 4 induces one of the following: a matching, a path, or a cycle. If we furthermore assume a maximum degree of at most 4 (which is not really a restriction in our context, see below), this knowledge of the local strucure enables us to show that our graph is either a line graph, or one of five graphs that resemble squares of cycles.

Lemma 2.1 (Structure lemma [3]) Let $G$ be a 3-connected claw-free graph with $\Delta(G) \leq 4$. If $G$ does not contain $K_{4}$ as $t$-minor then one of the following statements holds true:

(i) $G$ is a line graph;

(ii) $G \in\left\{C_{6}^{2}-v_{1} v_{6}, C_{7}^{2}-v_{7}, C_{10}^{2}-v_{10}, C_{7}^{2}, C_{10}^{2}\right\}$.

The natural next step is to examine the graphs from (ii) on $t$-perfectness. It turns out that the first three are $t$-perfect, and the vectors in $\mathbb{R}^{V\left(C_{i}^{2}\right)}$ with all entries $1 / 3$, which lie in $\operatorname{TSTAB}\left(C_{i}^{2}\right)$ but not in $\operatorname{SSP}\left(C_{i}^{2}\right)$, witness the fact 
that the latter two, as well as any square of a cycle of length at least 7, are $t$-imperfect.

Let us conclude the section with a remark on the maximum degree. If a claw-free graph $G$ has a vertex $v$ of degree at least 6 , then we can apply Ramsey's theorem on $N(v)$ to see that $G$ contains a $K_{4}$-subgraph. Similarly, a vertex of degree 5 leads to one of $K_{4}, W_{5}$ as an induced subgraph.

\subsection{Line graphs and colouring}

First of all, observe that a matching in a graph corresponds to a stable set of its line graph, and thus the SSP of a line graph is nothing else than the matching polytope $M(G)$ of the corresponding graph $G$. We can thus make use of Edmonds [7] celebrated theoremon the matching polytope. In fact, we only have to check that the inequalities given by (1) translate to those from Edmonds' theorem. This gives the following.

Lemma 2.2 [3] Let $H$ be a line graph (of a simple graph). Then $H$ is t-perfect if and only if $H$ does not contain $K_{4}$ as a $t$-minor.

It is now possible to use the knowledge on the structure of $H$ to find a colouring with three colours.

Lemma 2.3 [3] Let $H$ be a t-perfect line graph of a graph. Then $\chi(H) \leq 3$.

We use the lemma to prove our first main result.

Proof. [Proof of Theorem 2] We apply induction on the order of $G$. Observe that we may assume $G$ to be 2-connected (otherwise colourings of the blocks of $G$ can be combined). Now if $G$ is 3-connected, then we can apply Lemma 2.1. The possible outcomes of this lemma can either be coloured "by hand", or using Lemma 2.3.

On the other hand, if $G$ is not 3-connected, we find distinct vertices $u, v$, and induced proper subgraphs $L$ and $R$ of $G$ so that $V(L) \cap V(R)=\{u, v\}$ and $L \cup R=G$. As $|V(L)|,|V(R)|<|V(G)|$ there are, by induction, 3colourings $c_{L}$ and $c_{R}$ of $L$ and $R$. Permuting colours, if necessary, we may assume that $c_{L}(u)=c_{R}(u)$ and $c_{L}(v) \neq c_{R}(v)$ (if also $c_{L}(v)=c_{R}(v)$, we are done). Furthermore, we may assume that $c_{L}(u)=c_{L}(v)$.

Now if swapping the colours in $L$ on the Kempe-chain at $v$ of the colours $c_{L}(v)$ and $c_{R}(v)$ does not alter the colour of $u$ in $L$, we can thus combine our colourings of $L$ and $R$ to a colouring of $G$. Otherwise, there is an even $u-v$ path $P$ in $L$, which we can furthermore assume to be induced. Then, contracting $P$ while deleting the rest of $L$ we arrive at a $t$-minor $\tilde{R}$ of $G$ that 
is identical to $R$ with $u$ and $v$ identified. We apply induction to obtain a colouring of $\tilde{R}$ which together with $c_{L}$ yields a colouring of $G$.

\subsection{Characterising claw-free t-perfect graphs}

Lemma 2.1 together with Lemma 2.2 and the last paragraph of section 2.1 already provides a full characterisation of 3-connected claw-free $t$-perfect graphs. We shall show now that minimally $t$-imperfect claw-free graphs are, in fact, 3 -connected. This will be achieved by the combination of the following two lemmas. The first of these is very similar to Lemma 12 from Gerards and Shepherd [9], and the second builds on results of Barahona and Mahjoub [1] and Mahjoub [10].

Lemma 2.4 [3] Let $G$ be a minimally t-imperfect graph, and assume $u, v \in$ $V(G)$ to separate $G$. Then $G-\{u, v\}$ has exactly two components, one of which is a (possibly trivial) path. Moreover, uv $\notin E(G)$.

Lemma 2.5 [3] Let $G$ be a minimally t-imperfect claw-free graph. Then $G$ has minimum degree $\geq 3$.

We prove our second main result.

Proof. [Proof of Theorem 1] As neither of $K_{4}, W_{5}, C_{7}^{2}$ and $C_{10}^{2}$ is $t$-perfect, necessity is obvious. To prove sufficiency, consider a claw-free and minimally $t$-imperfect graph $G$. Lemmas 2.4 and 2.5 ensure that $G$ is 3-connected. Moreover, as we are done if $G$ contains $K_{4}$ or $W_{5}$ as a $t$-minor, it follows that $\Delta(G) \leq 4$. Consequently, we may assume that $G$ is of type (i) or (ii) as listed in Lemma 2.1. Now, if $G$ is a line graph then Lemma 2.2 forces $G$ to contain $K_{4}$ as a $t$-minor, as desired. On the other hand, as the graphs $C_{6}^{2}-v_{1} v_{6}$, $C_{7}^{2}-v_{7}$ and $C_{10}^{2}-v_{10}$ are $t$-perfect, it follows that $G \in\left\{C_{7}^{2}, C_{10}^{2}\right\}$, as desired.

\section{References}

[1] F. Barahona and A. R. Mahjoub, Compositions of graphs and polyhedra II: stable sets, SIAM J. Discrete Math. 7 (1994), 359-371.

[2] M. Boulala and J.P. Uhry, Polytope des indépendants d'un graphe sérieparallèle, Disc. Math. 27 (1979), 225-243.

[3] H. Bruhn and M. Stein, On claw-free t-perfect graphs, submitted.

[4] M. Chudnovsky and A. Ovetsky, Coloring quasi-line graphs, J. Combin. Theory (Series B) 54 (2007), 41-50. 
[5] M. Chudnovsky, R. Seymour, N. Robertson, and R. Thomas, The strong perfect graph theorem, Ann. Math. 164 (2006), 51-229.

[6] V. Chvátal, On certain polytopes associated with graphs, J. Combin. Theory (Series B) 18 (1975), 138-154.

[7] J. Edmonds, Maximum matching and a polyhedron with 0,1-vertices, J. Res. Natl. Bur. Stand., Sect. B 69 (1965), 125-130.

[8] J. Fonlupt and J.P. Uhry, Transformations which preserve perfectness and $h$ perfectness of graphs, Ann. Disc. Math. 16 (1982), 83-95.

[9] A.M.H. Gerards and F.B. Shepherd, The graphs with all subgraps t-perfect, SIAM J. Discrete Math. 11 (1998), 524-545.

[10] A. R. Mahjoub, On the stable set polytope of a series-parallel graph, Math. Programming 40 (1988), 53-57.

[11] N. Sbihi and J.P. Uhry, A class of h-perfect graphs, Disc. Math. 51 (1984), 191-205.

[12] A. Schrijver, Combinatorial optimization. Polyhedra and efficiency, SpringerVerlag, 2003. 